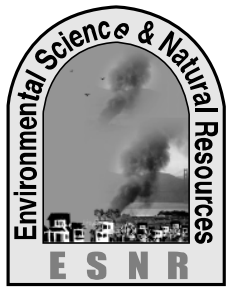

J. Environ. Sci. \& Natural Resources, 6(1): 253 - 259, 2013

ISSN 1999-7361

\title{
Investigation on the Nitrogen Fixing Cyanobacteria (BGA) in Rice Fields of North-West Region of Bangladesh. III: Filamentous (Heterocystous)
}

\author{
M. A. Hasan \\ Department of Agronomy and Agricultural Extension, Rajshahi University, Bangladesh
}

\begin{abstract}
The experiment was conducted to carry out the nitrogen fixing cyanobacterial (BGA) resources and their distribution pattern in rice fields of North-West region of Bangladesh during the period of 2011. Considering the vast importance of Blue-green algae (BGA) in agricultural land especially in rice fields, their twelve months long systematic was undertaken. A total of 10 genera with 37 species of filamentous-heterocystous blue-green algal forms were identified and recorded from this region. Out of 37 species, 9 species belonging to the genus Anabaena, 8 to Nostoc, 5 to Cylindrospermum, 4 to Calothrix, 3 to Rivularia, 3 to Gloeotrichia and 2 to Aulosira; while 1 species belonging to each genus of Anabaenopsis, Microcheate and Aphanizomenon. The distribution pattern of filamentous-heterocystous BGA in rice fields over the study area has also been observed in this study. With few exceptions, the filamentous-heterocystous BGA are more or less uniformly distributed all over the study areas. All of the BGA members have a tremendous ability to contribute on enhancing agricultural production by fixing atmospheric nitrogen and adding organic matter, vitamins, growth promoting substances etc to the soil and crops.
\end{abstract}

Keywords: Bangladesh, Filamentous-heterocystous, Nitrogen fixing cyanobacteria (BGA), North-West Region, Rice fields.

\section{Introduction}

Bangladesh is an agriculture base country with ever-growing population. Competing to the time, the demand of agricultural products is being increased significantly. Moreover, most of the farmers of Bangladesh are marginal and of low living standard. It is difficult to them to use highly cost and problem inducing chemical fertilizers. To meet the challenge of ever increasing food demand and enhance the living standard of our farmers, there is no alternative but increase the crop production by low production cost. Blue-green algae especially their bio-fertilizers are the right ones for meeting this challenge as they are capable to increase crop yield in $10-15 \%$ by fixing $20-30$ $\mathrm{Kg}$ N/ha. Cyanobacteria (Blue-Green Algae) are one of the major components of the nitrogen fixing biomass in paddy fields. The agricultural importance of cyanobacteria in rice cultivation is directly related with their ability to fix nitrogen and other positive effects for plants and soil. After water, nitrogen is the second limiting factor for plant growth in many fields and deficiency of this element is met by fertilizers (Malik et al., 2001). The excessive use of chemical fertilizers has generated several environmental problems including the greenhouse effect, ozone layer depletion and acidification of soil and water. These problems can be tackled by use of biofertilizers (Choudhury and Kennedy 2005; Rai, 2006). Bio-fertilizers, more commonly known as microbial inoculants, include bacteria (Rhizobium, Azotobacter etc.), algae (Blue-green algae) and mycorrhizal fungi; they are natural, beneficial and ecological, and they provide nutrients for the plants and maintain soil structure (Board 2004). Specially, cyanobacteria (BGA) along play an important role in maintenance and build-up of soil fertility, consequently increasing rice growth and yield as a natural bio-fertilizer (Song et al., 2005). The acts of these algae include: (1) they converted huge amount of $\mathrm{CO}_{2}$ into $\mathrm{O}_{2}$ as they bear photosynthetic pigments and thus putting a vital role in repairing environmental pollution. (2) Increase in soil pores and production of adhesive substances (3) the same way, they enhance the soil aeration results in supplying a considerable amount of $\mathrm{O}_{2}$ to the crop rhizosphere. (4) Excretion of growth-promoting substances such as hormones (auxin, gibberellins), vitamins, amino acids (Roger and Reynaud, 1982, Rodriguez et al., 2006). (5) Increase in water-holding capacity through their jelly structure (Roger and Reynaud, 1982). (6) Increase in soil biomass after their death and decomposition. (7) Decrease in soil salinity. (8) Preventing weeds growth. (9) Increase in soil phosphate by excretion of organic acids (Wilson 2006). Most paddy soils have a natural population of cyanobacteria which provides a potential source of nitrogen fixation at no cost (Mishra and Pabbi, 2004). Considering the tremendous important of BGA, it should be the first priority to search out and identify this resources and their distribution pattern in our rice fields. Only then, they may be used in producing algal bio-fertilizer, enhancing soil fertility and rice production, reducing chemical fertilizer utilization and production cost, ensuring friendly environment to agriculture. Against this backdrop, this study has been undertaken.

Therefore, the present paper deals with: i. Search out the filamentous-hetrocystous members of bluegreen algal resources in the rice fields of NorthWest region of Bangladesh, ii. Find out their distribution pattern over the same. [Amongst BGA, this paper is concerned only with filamentousheterocystous members.] 


\section{Materials and Method}

Five districts viz. Serajgonj, Pabna, Natore. Rajshahi and Naogoan of North-West part of Bangladesh have been considered as study zone. Rice field of two upozillas of each district like as Ullapara and Tarash of Serajgonj, Chatmohore and Ishwardi of Pabna, Gurudashpur and Singra of Natore, Rajshahi proper and Godagari of Rajshahi and Atri and Patnitala of Naogaon district have been selected as sampling spot. In all sampling spots spread over the study zone were selected for the collection of blue-green algal sample. The climatological and ecological variations have been intensively considered during the spot selection.

Sampling was made at monthly interval from ten study spots. Commonly confined the surface layers, soil algal samples were collected by means of a diminutive rectangular shovel having a sharp blade. While the study field was damp, a small block could be removed directly by cutting with a scalpel. It is often difficult to separate algal specimen from soil particles. To obtain relatively clean samples, the soil blocks, were kept in a petri-dish and added enough water to saturate them. A few cover glasses were placed on the soil block and the dish was left open until the excess water had evaporated. Within in one or two days, motile algae in the soil crept up on the underside of the cover glasses and began to multiply. According to necessity, the samples were preserved by spraying Transeau's solution on the petri-dish. A drop of $10 \%$ glycerin was placed on a clean slide and the algae contain cover slip was mounted on it and examined under the microscope. During the microscopic observation, only the BGA members are considered to be the desired specimen for the present study. Three slides of each sample have been observed under microscope to ensure the appearance or disappearance of any blue-green algal genus and species under themselves and also to confirm their distribution pattern. The drawing of blue-green algal specimen was done by using Camera Lucida. The measurement and microscopic calibration is done usually by the ocular micrometer which consists of an occulometer or eye piece micrometer and a stage micrometer. The systematic identification was done with the help of standard works by Dsikachary (1959), Pandey (1965), Islam (1967, 1973 and 1981).

\section{Results and Discussion}

\section{Taxonomy}

In all 10 genera with 37 species of cyanobacterial filamentous-heterocystous taxa have been described systematically and illustrated in this paper. Out of 37 species, 9 species belonging to the genus Anabaena, 8 to Nostoc, 5 to
Cylindrospermum, 4 to Calothrix, 3 to Rivularia, 3 to Gloeotrichia and 2 to Aulosira; while 1 species belonging to each genus of Anabaenopsis, Microcheate and Aphanizomenon. However, 37 species under 10 genera are plotted in two plates viz. plate- 1 and plate- 2 . [All the measurements are expressed in $\mu$ ].

\section{CLASS: CYANOPHYCEAE ORDER: NOSTOCALES FAMILY: NOSTOCACEAE}

\section{Anabaena anomala Fritsch (Pl. 1, Fig. 9)}

Desikachary, 1959, 398, Pl.. 73, Fig. 2

Thallus very thin, gelatinous, expanded on moist soil, blackish blue-green; trichomes many, entangled, less contorted, with distinct thin confluent sheath, moniliform; vegetative cells 4.26.2 diam., 6.6-13.8 long; heterocyst intercalary, sub-spherical, 5.6-9.9 long, 6.6 diam.; end cell sub-conical, obtuse.

\section{Anabaena spherica var. attenuata} Bharadwaja (Pl. 1, Fig. 19)

Desikachary, 1959, 393, Pl. 71, Fig. 8

Thallus gelatinous, thin, pale blue-green, trichome entangled with each other, curved or straight, attenuated at the ends, cell loosely attached, constricted at the cross walls, 6.0-8.5 long, 3.5-4.6 diam.; heterocyst more or less spherical, 5.0-7.5 diam., with smooth wall, spore in series, spherical to oval, 6.0-9.9 long, 5.0-7.9 diam.

3. Anabaena naviculoides Fritsch (PI. 1, Fig. 17) Desikachary, 1959, 410, Pl. 72, Fig. 2; Pandey, 1965, 197, Pl. 6, Fig. 1; Pl. 12, Fig. 2

Plant mass gelatinous, Trichome elongate, contorted; cells 3.0-4.0, diam., 3.3-5.0 long, barrel shaped, apical cell conical, obtuse; heterocyst intercalary, 6.0-7.9 diam., 5.0-6.5 long; akinete ellipsoidal, in series, loosely attached, 4.5-6.0 diam., 12.8 long, exospores hyaline.

\section{Anabaena oryzae Fritsch (Pl. 1, Fig. 2)}

Desikachary, 1959, 396, Pl. 72, Fig. 3

Thallus soft, green, gelatinous, membranous, trichome short, straight, densely arranged, generally parallel, 3.0-3.8 broad, more or less barrel shaped, 1.5 to 2 times as long as broad, heterocyst terminal and intercalary, broader than the vegetative cells, 3.3-4.5 broad, spores rarely single to the terminal heterocyst, commonly away from the intercalary heterocyst, single or 2-7 in series, sub-spherical or shorter ellipsoidal, 4.5-4.9 long, 5.0-5.3 diam.

\section{Anabaena volzii Lemm. (Pl. 1, Fig. 14)}

Desikachary, 1959, 403, Pl. 77, Fig. 1; Islam, 1973, 135, Pl. 2, Figs. 27-29

Trichome solitary, straight; cells cylindrical, constricted at the cross walls3.5-6.5 long, 3.4-3.6 diam.; heterocyst cylindrical, 6.5 diam., 8.5 long. 
6. Anabaena fertilissima Rao, C. B. (PI. 1, Fig. 13) Desikachary, 1959, 398, Pl. 74, Fig. 1

Trichome solitary, mostly straight, slightly bent, end cell with rounded ends; vegetative cells 3.8-6.0 diam., 3.3 long; heterocyst intercalary, 6.6 diam., 6.5-8.5 long.

7. Anabaena variabilis var. ellipsospora Fritsch (Pl. 1, Fig. 6)

Desikachary, 1959, 411, Pl. 72, Fig. 1

Thallus thin gelatinous, brownish blue-green; trichomes straight or slightly flexuous, loosely arranged, constricted at the cross walls; apical cells rounded, sometimes obtuse; cells spherical or barrel shaped, 4.0-6.4 broad, 6.5-9.4 long in series away from heterocyst; heterocyst intercalary, ellipsoidal, 4.5-6.5 diam., 8.5-15.5 long.

8. Anabaena circinalis Rabenhorst ex. Born. et. Flah. (Pl. 2, Fig. 18)

Desikachary, 1959, 414, Pl. 77, Fig. 2; Yamagishi \& Hirano, 1973, 78, Pl. 7, Fig. 21; Pl. 8, Figs. 2, 5

Trichome mostly coiled, circinate, without prominent sheath, cells spherical to barrel shaped, 3.8-6.9 diam., 3.5-5.3 long; heterocyst many, intercalary, spherical to ellipsoidal, 5.0-9.9 long, 4.0-5.0 diam.; akinete ellipsoidal to cylindrical, 7.2-8.5 diam., 14.5-18.0 long, away from the heterocyst; epispore smooth and colorless.

\section{Anabaena circinalis var. crassa Ghose (PI. 2, Fig. 1)}

Desikachary, 1959, 412, Pl. 77, Fig. 5

Trichome free-swimming, single, semi-circular, loosely coiled up to 4 times; cells nearly spherical but generally shorter than broad, 5.4-6.6 in diam. with pseudo-vacuole; heterocyst globose, 7.2 diam.; spore not seen.

10. Anabaenopsis circularis var. javanica Wolosz (Pl. 1, Fig. 15)

Desikachary, 1959, 356, Pl. 63, Figs. 3, 5

Trichome short, spirally coiled, fragile; cells spherical or somewhat longer than broad; 3.3-5.0 long, 3.5 diam.; heterocyst intercalary, spherical, 4.3-6.0 diam.; spore absent.

\section{Aphanizomenon flos-aquae (Linn.) Ralfs ex Born. et. Flah. (PI. 1, Fig. 3)}

Desikachary, 1959, 359, Pl. 64, Fig. 8

Trichome in bundles, sometimes solitary with other algae, mostly straight, apices slightly bent; cells 4.57.6 diam., 4.5-10.8 long; heterocyst 4.8-7.5 diam., 16.5-24.0 long; akinete single, nearly median away from the heterocyst, cylindrical, 5.1-8.8 diam., 40.052.5 long, epispore smooth and colorless.

12. Nostoc calcicola Brebisson ex Born. et. Flah.(Pl. 1, Fig. 16)

Desikachary, 1959, 384, Pl. 68, Fig. 1

Thallus pale olive green, diffluent, filaments loosely entangled, sheath indistinct, sometimes partly distinct, hyaline; cells spherical to barrel shaped, blue-green, 3.3-5.0 long, 4.0-5.2 diam.; heterocyst one or more, intercalary, sub-spherical, 4.2-6.0 diam.

13. Nostoc commune Vaucher ex Born. et Flah. (Pl. 1, Figs. 10)

Desikachary, 1959, 387, Pl. 68, Fig. 3

Thallus firm and gelatinous, bluish-green, irregular shaped; filaments entangled; sheath hyaline; vegetative cells barrel shaped, sometimes spherical, 3.55 long, 3.3-5.0 diam., heterocyst terminal and intercalary, 4.7-7.5 diam., spore not found.

14. Nostoc muscorum Ag. ex. Born. et Flah. [PI. 1, Figs. 11 (a-b)]

Desikachary, 1959, 385, Pl. 70, Figs. 2 \& 4

Thallus gelatinous, flat, expanded on soil, brownish blue-green; filaments entangled; sheath not distinct; trichome long, 3.3 diam., constricted at the cross wall; cells spherical barrel shaped, 4.87.0 long; heterocyst more than one, intercalary, spherical to barrel shaped, 6.6-10.0 long, 4.0-5.5 diam.; spores in series, 6.6-9.9 long, 5.5 diam., epispore yellowish.

15. Nostoc paludosum Kützing ex. Born. et Flah. (Pl. 1, Figs. 18)

Desikachary, 1959, 375, Pl. 69, Fig. 2; Komarek, 1975, 611, Pl. 6, Figs. 1-2

Thallus gelatinous, sheath broad, yellowish brown, filaments centered; Trichome 4.0-5.5 broad; cells barrel shaped, as long as broad; pale blue-green; heterocyst terminal, sometimes intercalary, 5.5-6.8 broad, 6.0-7.5 long; spores in series, 3.8-5 broad, 6.5 long.

\section{Nostoc hatei Dixit(Pl. 1, Fig. 12)}

Desikachary, 1959, 383, Pl. 67, Fig. 2

Thallus spherical, filaments densely entangled, irregularly curved or contorted; trichome 4.0-7.0 diam.; cells spherical, as long as broad; heterocyst single or in series; spherical, 4.5-8.0 long, 3.8-7.2 diam.

17. Nostoc linkia (Roth.) Bornet ex. Born. et Flah. (Pl. 2, Fig. 2)

Pandey, 1965, 196, Pl. 5, Fig. 3

Thallus of various size, tuberculate, irregularly expanded when old, blue-green, later blakish green; filaments densely entangled, much contorted; trichome deeply constricted, appear loosely connected; cells more or less barrel shaped, 4.0-6.5 long, 4.0-5.0 diam.; heterocyst sub-spherical, 4.0-6.5 diam., 6.5 long.

18. Nostoc carneum Ag. ex. Born. et Flah. (PI. 2, Fig. 3) Islam and Begum, 1981, 3, Pl. 3, Figs. 16-17

Trichome long, slightly constricted, light bluegreen, trichome and heterocyst small in diameter; cells 4.7-5.0 long, 2.2-2.5 broad; heterocyst cylindrical, 5.5-6.0 long, 3.0-3.3 broad. 


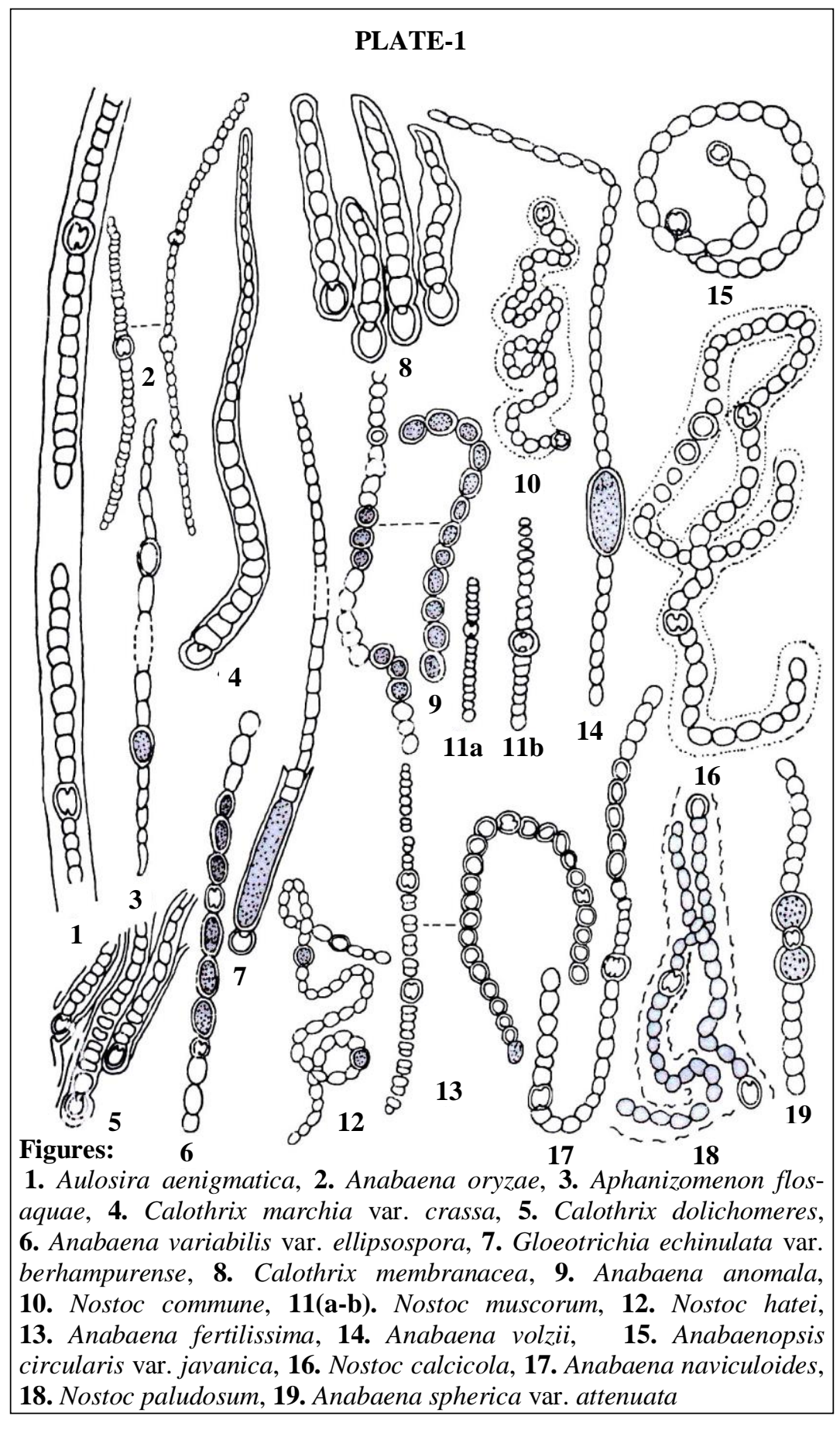

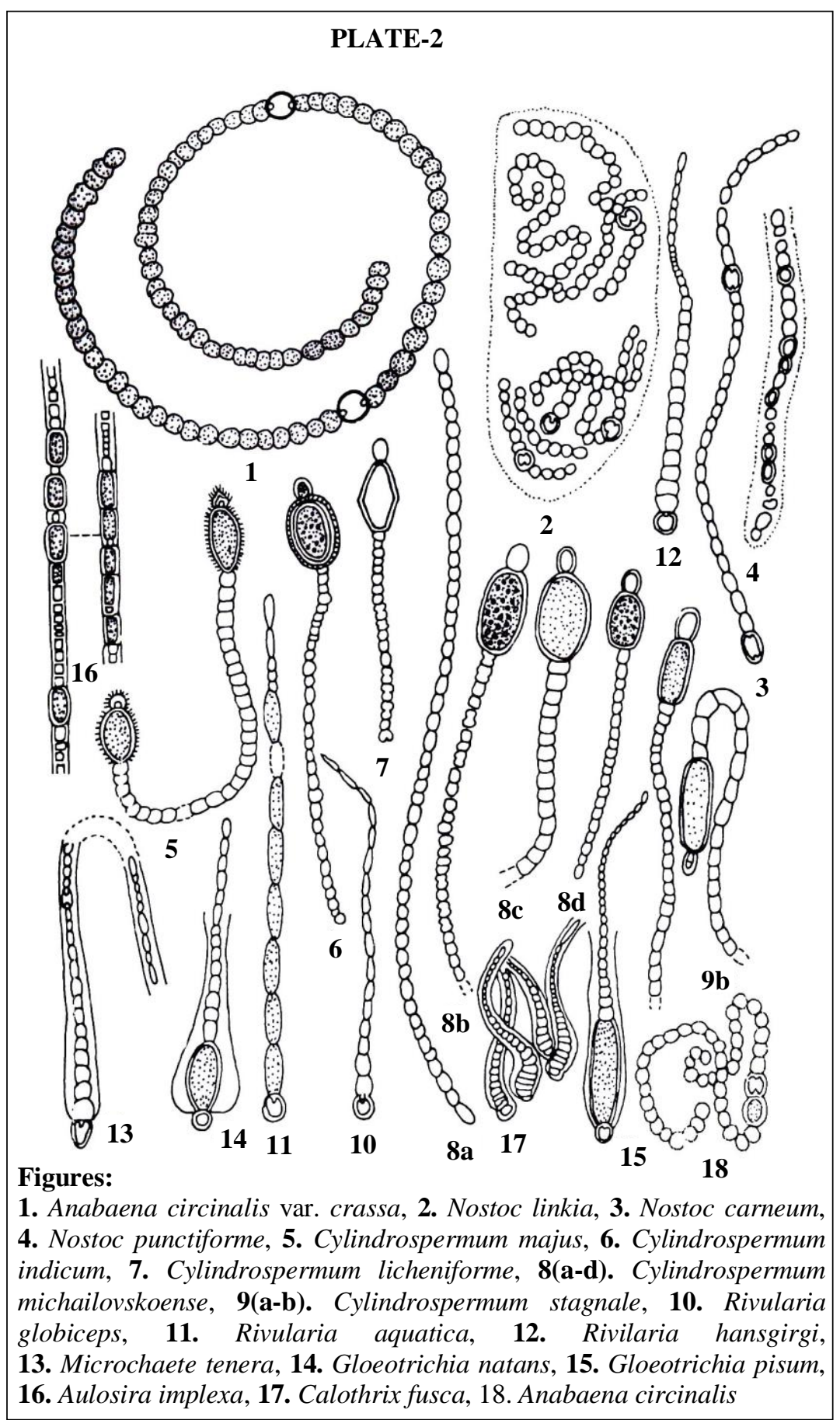


19. Nostoc punctiforme (Kütz.) Hariot (Pl. 2, Fig. 4) Desikachary, 1959, 374, Pl. 69, Fig. 1; Pandey, 1965, 195, Pl. 4, Fig. 7 \& Pl. 11, Fig. 5

Thallus globose; Filaments flexuous, densely entangled with each other; sheath hyaline to slightly brownish; trichome 3.8-6.0 diam, constricted at the cross walls; cells sub-spherical to barrel shaped, 5.07.5 long; heterocyst 4.5-6.4 diam., 6.0-7.8 long; spores in series.

\section{Aulosira aenigmatica Femy (Pl. 1, Fig. 1)}

Desikachary, 1959, 428, Pl. 81, Figs. 15, 17

Filaments intricate; sheath close, colorless, thin; trichome pale blue-green; cells 4.9-5.1 broad, 6.6 long, apical cell broadly conical; heterocyst rectangular, single, as long as broad or slightly longer.

21. Aulosira implexa Bonet et Flahault (PI. 2, Fig. 16) Islam, 1973, 136, Pl. 13, Figs. 23-24; Pandey, 1965, 198, Pl. 6, Fig. 4

Filaments straight, 12.8-15.0 diam.; sheath prominent, thin, hyaline, unlamellated, slightly brownish; trichome slightly constricted at the cross walls; cells quadrate, 8.610 .0 diam., 6.8- 15.0 long; heterocyst quadrate to oblong; 6.6-19.5 long, 5.0-9.9 diam.

22. Cylindrospermum majus Kützing ex. Born. et Flah. (Pl. 2, Fig. 5)

Desikachary, 1959, 360, Pl. 80, Fig. 1

Thallus mucilaginous, brownish to yellowish green in color; trichome irregularly contorted, mostly entangled, 4.0-6.6 diam., constricted at the cross walls; cells 3.65.6 long, cylindrical to quadrate, 4.2-6.5 diam.; heterocyst at both ends, 6.0-7.2 long, 4.0-6.5 diam., with papillae (many cilia like projections); akinetes at both ends immediately after heterocysts, ellipsoidal, 12.4-22.5 long, 9.5-14.6 diam with papillae.

23. Cylindrospermum indicum Rao, C. B. (Pl. 2, Fig. 6) Desikachary, 1959, 369, Pl. 64, Figs. 4, 11

Trichome single with deep constrictions at the joints, 3.6 broad, dark blue-green; cells almost quadrate, or more or less barrel shaped, 3.3-4.7 long; heterocysts spherical or sub-spherical, sub-conical or ellipsoidal, sometimes cylindrical, one at each end of the trichome, 3.0-5.5 broad and 3.3-7.0 long; spores almost elliptical, without membrane $8.5-9.3$ broad and with membrane 9.9-12.0 broad, without membrane 15.0-17.5 long, with membrane 17.0-22.0 long.

\section{Cylindrospermum licheniforme Kütz ex. Born.} et Flah. (Pl. 2, Fig. 7)

Desikachary, 1959, 366, Pl. 65, Fig. 8

Thallus mucilaginous, orbicular, confluent, later more or less expanded, blackish green; trichome 3.3-4.5 broad, constricted at the cross walls, pale blue-green; cells quadrate to cylindrical, 4.2-5.5 long; heterocyst oblong, 5.5-6.6 broad, 6.6-9.9 long; spores oblong to ventricose elliptic, 10.5-14.0 broad, 19.7-25.5 long.

\section{Cylindrospermum michailovskoense Elenkin [PI. 2, Figs. 8(a-d)]}

Desikachary, 1959, 368, Pl. 65, Fig. 1

Thallus expanded, dull blue-green; trichome pale blue-green, constricted at the cross walls, 7.0-10.0 diam.; cells 9.6-16.5 long; heterocysts at both ends, spherical, 9.0-10.3 diam.; akinetes at both ends immediately after the heterocyst, also intercalary, ellipsoidal, 9.9 diam., 15.5-19.2 long.

26. Cylindrospermum stagnale (Kütz.) Born. et Flah. [PI. 2, Figs. 9(a-b)]

Desikachary, 1959, 363, Pl. 65, Fig. 9

Thallus expanded, blue-green; trichome 4.0-6.4 broad, constricted at the cross walls; cells nearly quadrate; heterocyst terminal, subspherical to cylindrical, 9.0-11.3 diam., 20.0-22.8 long; akinetes cylindrical, up to 40.0 long, $12.5 \mathrm{diam}$.

\section{ORDER: NOSTOCALES}

\section{FAMILY: RIVULARIACEAE}

27. Rivularia globiceps West, G. S. (PI. 2, Fig. 10) Desikachary, 1959, 552; Islam, 1973, 131, Pl. 2, Figs. 3-4; Pl. 3, Figs. 15-18

Thallus small, soft, spherical, filaments closely ad pressed, constricted at the cross walls; sheath colorless, unlamellated; trichome gradually attenuated and drawn out into a long hair; basal cells 6.0-9.0 long, 4.5-7.5 diam.; heterocyst terminal, single, hemispherical, 8.2-12.5 diam.

\section{Rivularia aquatica De Wilde (PI. 2, Fig. 11)}

Desikachary, 1959, 552, Pl. 116, Figs. 2-3

Thallus small, spherical, soft, filaments slightly ad pressed together; sheath very thin, colorless, not lamellate; trichome gradually attenuated at the end, 6.6 diam.; cells at the base loger than broad, 6.0-10.0 long, 6.5-7.3 diam.; terminal cells drown out into a long colorless hair.

\section{Rivilaria hansgirgi Schmidle (PI. 2, Fig. 12)}

Desikachary, 1959, 549, Pl. 112, Fig. 7

Thallus expanded, flat, thin, gelatinous, blackish brown; trichome long, curved, gradually attenuated, 6.8 broad at the middle, 3.3 broad at the apices; cells rectangular or sub-quadrate at the basal region, broader than long, 5.5-6.6 diam., 4.0-5.0 long; heterocyst basal, single, subspherical or sometimes hemispherical, 8.5-9.0 diam.

30. Gloeotrichia echinulata var. berhampurense Rao, C. B (Pl. 1, Fig. 7)

Desikachary, 1959, 558, Pl. 118, Figs. 12-13

Thallus hard, small, up to $3 \mathrm{~mm}$. in diam.; filaments radiating regularly from the center; sheath prominent, unlamellate, slightly diffluent in mature filaments, hyaline; trichomes attenuated at the end into a long hair; cells at the base 4.9-5.2 
long, 6.5 diam.; heterocyst single, terminal, sub-spherical, 9.5 diam.; akinete next to the heterocyst, cylindrical smooth walled, 9.0-11.2 diam., 70.0-94.5 long.

31. Gloeotrichia natans Rabenh. ex. Born. et Flah. (Pl. 2, Fig. 14)

Desikachary, 1959, 561, Pl. 118, Figs. 7, 15

Thallus spherical, soft, blackish green; filaments loosely arranged, olive-green; trichome gradually attenuated into a long hyaline hair; sheath prominent, diffluent, saccade, 10.7-13.5 diam.; vegetative cells at the base 9.0-11.3 long, 11.0-13.5 diam; heterocyst terminal, spherical, 9.9-12.2 diam.; akinete 9.0-10.0 diam., 51.0-79.0 long.

32. Gloeotrichia pisum Thuret ex. Born. et Flah. (Pl. 2, Fig. 15)

Desikachary, 1959, 555, Pl. 116, Figs. 4-5; Geitler, 1925, 231, Figs, 34 \& 278

Thallus very small, spherical, $1.51 \mathrm{~mm}$. in diam., dull blue-green; filaments densely entangled, ad pressed; sheath prominent, colorless; trichome gradually attenuated, drawn out into a long hair; vegetative cells 9.0-12.8 long, 5.5-8.5 diam. at the basal region; terminal cells 19.0-21.0 long, 3.3-4.0 diam.; basal heterocyst spherical, 9.0-13.5 diam.; akinete next to the heterocyst, cylindrical, up to 13.5 diam., 81.0-97.5 long.

\section{Calothrix dolichomeres Skuja(PI. 1, Fig. 5)}

Desikachary, 1959, 540, Pl. 109, Figs.1-5

Filaments erect and slightly curved, aggregate, false branched in pairs or single; sheath thin, lamellate, colorless, diffluent, about 2.3 thick; basal cells 6.6-9.5 broad, 6.5-12.8 long, constricted at the cross walls; heterocyst basal, rounded or sometimes cylindrical, 7.8-9.5 broad, 9.2-11.5 long.

34. Calothrix marchia var. crassa Rao, C. B. (PI. 1, Fig. 4) Desikachary, 1959, 534, Pl. 113, Figs. 3-4; Islam, 1973, 128, Pl. 3, Figs. 14-15

Thallus blue-green; filaments bent; sheath thin, firm, tough, hyaline, unlamellate, covering the entire trichome; trichome gradually attenuated; cells 3.3-5.5 long, 9.0-10.5 diam. at the base; heterocyst basal, sub-spherical, 5.0-6.5 long, 9.0-10.5 diam.

35. Calothrix membranacea Schmidle (PI. 1, Fig. 8) Desikachary, 1959, 542, Pl. 106, Fig. 10

Thallus thin, papery, blue-green; filaments long, erect, curved, forming irregular mass, sparingly branched, attenuated at the end; sheath thin, firm, hyaline, unlamellate; trichome 3.6-6.5 broad; cells sub-quadrate, 5.6 long; heterocyst basal, 6.7 broad, as broad as long.

36. Calothrix fusca (Kütz.) Bornet et Flah. (PI. 2, Fig. 17) Desikachary, 1959, 527, Pl. 107, Fig. 10; Islam, 1973, 128, Pl. 3, Figs. 4-8
Filaments few in groups or in single, rarely bent and inflated; sheath thick, broad, colorless, lamellate, diffluent at the upper end; vegetative cells at the basal region 7.0-15.5 diam., 9.5 long; heterocyst basal, spherical to hemispherical, 9.8 long, 8.5 diam.; terminal cells of the trichome drawn out into a long hair.

\section{ORDER: NOSTOCALES FAMILY: MICROCHAETACEAE}

37. Microchaete tenera Thuret ex. Born. et Flah. (Pl. 2, Fig. 13)

Desikachary, 1959, 513, Pl. 105, Figs. 1, 2

Filament single or in clusters; slightly bent at the basal region, 7.5 diam.; sheath thin, unlamellate, hyaline; trichome 4.0-6.5 diam.; basal cells longer than broad, 6.4-7.5 long; heterocyst basal or intercalary, spherical, sometimes cylindrical, spherical heterocyst 4.5 diam., cylindrical heterocyst 4.5 diam., 7.2-11.3 long; spore intercalary, cylindrical.

\section{Distribution of Cyanobacteria (BGA)}

Out of 10 genera, Anabaena, Nostoc, and Cylindrospermum are distributed all over the study region with higher abundant; while Calothrix, Rivularia, Gloeotrichia and Aulosira are distributed with moderately abundant. Contrary to this, Anabaenopsis, Microcheate and Aphanizomenon are distributed all over the study areas with less abundant. Anabaenopsis is not found in the rice fields of Pabna and Naongoan district; while Microcheate is not observed only in Rajshai district (Table. 1).

A profusely distribution of cyanobacteria (BGA) is observed in the rice fields of Serajgonj district followed by Natore and Pabna. All the members of BGA are more or less uniformly distributed in these areas. Probably, it may due to the medium to high soil organic matter content and higher soil fertility status of these areas which favor the usual growth of cyanobacteria. On the other hand, comparatively a poor and slightly fluctuated distribution of cyanobacteria is found in the rice fields of Rajshahi and Naogoan district. Such findings probably due to the medium to low soil organic matter content with low moisture holding capacity and lower soil fertility status of these areas which may adversely affect on the usual growth of cyanobacteria as the upper part of both the Rajshahi and Naogoan district are in level, level and high Barind-Tract atmosphere, respectively. However, with few exceptions, more or less a uniform distribution of filamentous-heterocystous cyanobacteria (BGA) has been found all over the study region. 
Table 1. The distribution of Cyanobacteria (filamentous-heterocystous) in rice fields of North-West region of Bangladesh

\begin{tabular}{|c|c|c|c|c|c|}
\hline \multirow[t]{2}{*}{ Genera } & \multicolumn{5}{|c|}{ Mode of Existence } \\
\hline & Serajgonj & Pabna & Natore & Rajshahi & Naogoan \\
\hline Anabaena & +++ & +++ & +++ & +++ & +++ \\
\hline Nostoc & +++ & ++ & +++ & ++ & ++ \\
\hline Cylindrospermum & +++ & +++ & +++ & ++ & ++ \\
\hline Calothrix & ++ & ++ & ++ & ++ & + \\
\hline Rivularia & ++ & ++ & +++ & ++ & ++ \\
\hline Gloeotrichia & +++ & ++ & ++ & + & ++ \\
\hline Aulosira & ++ & ++ & ++ & + & + \\
\hline Anabaenopsis & + & - & + & + & - \\
\hline Microcheate & ++ & ++ & + & - & + \\
\hline Aphanizomenon & ++ & + & ++ & + & + \\
\hline
\end{tabular}

$+=$ Less abundant, $++=$ Moderately abundant, $+++=$ Highly abundant, $-=$ Absent

\section{Conclusion}

The present investigation notices that the nitrogen fixing filamentous-heterocystous Cyanobacteria (BGA) is more or less uniformly distributed all over the study region with few exceptions. However, in the present study, 37 nitrogen fixing filamentous-heterocystous cyanobacterial (BGA) specimens under 10 genera are introduced to us which may be used in producing algal bio-fertilizer, enhancing soil fertility and rice production, reducing chemical fertilizer utilization and production cost, ensuring friendly environment to agriculture as they are capable to fix atmospheric nitrogen by no cost and produce organic manures, vitamins and growth promoting substances naturally.

\section{References}

Board, N. 2004. The Complete Technology Book on Biofertilizer and Organic Farming, New Delhi

Choudhury, A. T. M. A. and Kennedy, I. R. 2005. Nitrogen fertilizer losses from rice soils and control of environmental pollution problems. Communications in Soil Science and Plant Analysis, 36: 1625-1639.

Desikachary, T. V. 1959. Cyanophyta. Indian Council of Agricultural Research (ICAR). New Delhi, India. p.686.

Geitler, L. 1925. In Pascheris Diesusswassar flora, Heft 12. Cyanophyceae Verlag V. Gustar Fisher, Jena.

Islam, A. K.; Nurul, M. and Uddin, M. A. 1973. Fresh water algae of Bangladesh. II. Cyanophyceae. Dacca Univ. Stud. B., 21(2): 127-132.

Islam, A. K.; Nurul, M. and Nahar, L. 1967. Ibid., ibid. 4(2-3): 141-149.

Islam, A. K. and Nurul, M. 1973. Fresh water algae of Bangladesh. III. Cyanophycae. Dacca Univ. Stud. B., 21(2): 133-138.
Islam, A. K.; Nurul, M. and Begum, Z. T. 1981. Addition to the list of blue-green algae of Bangladesh. I. Dhaka Univ. Stud. B., 29(1): 49-57.

Komarek, J. 1975. Blaualgen aus dem naturschutzgebiet Rayice. Nova Hedwigia, XXVI (2-3): 636-644.

Malik, F. R., Ahmed, S. and Rizki, Y. M. 2001. Utilization of lignocellulosic waste for the preparation of nitrogenous biofertilizer. Pakistan Journal of Biological Sciences, 4: 12171220.

Mishra, U. and Pabbi, S. 2004. Cyanobacteria: a potential biofertilizer for rice. Resonance, 6-10.

Pandey, D. C. 1965. A study on the algae of paddy-field soils of Ballia and Gazipur districts of Uttar Pradesh. India. Part II (A): Taxonomic considerationCyanophyceae. Nova Hedwigia, X(I):77-209.

Rai, M. K. 2006. Handbook of Microbial Biofertilizers. Haworth Press, New York.

Roger, P. A. and Reynaud, P. A. 1982. Free-living Blue-green Algae in Tropical Soils. Martinus ijhoff Publisher, La Hague.

Rodriguez, A. A.; Stella, A. A.; Storni, M. M.; Zulpa, G. and Zaccaro, M. C. 2006. Effects of cyanobacterial extracellular products and gibberellic acid on salinity tolerance in Oryza sativa L. Saline System, 2: 7

Song, T.; Martensson, L.; Eriksson, T.; Zheng, W. and Rasmussen, U. 2005. Biodiversity and seasonal variation of the cyanobacterial assemblage in a rice paddy field in Fujian, China. The Federation of European Materials Societies Microbiology Ecology, 54: 131-140.

Wilson, L. T. 2006. Cyanobacteria: A Potential Nitrogen Source in Rice Fields. Texas Rice, 6: 9-10.

Yamagishi, T. and Mirano Hirano. 1973. Some fresh water algae from Cambodia. Contrib. Biol. Lab. Kyoto University, Japan. 24(2): 61-85. 